\title{
DIFFERENTIAL PARAMETERS OF THE FIRST ORDER*
}

BY

\section{HEINRICH MASCHKE}

The general expression of a differential parameter of the first order is given in the symbolic representation + by

$$
\left(U^{\prime} \ldots U^{\lambda} f^{\lambda} \cdots f^{n-\lambda}\right)\left(V^{\prime} \ldots V^{\lambda} f^{\prime} \cdots f^{n-\lambda}\right)
$$

where $U^{\prime}, \ldots, U^{\lambda}$ and $V^{\prime}, \ldots, V^{\lambda}$ are functions of $x_{1}, \cdots, x_{n}$, and $f^{\prime}, \cdots, f^{n-\lambda}$ are equivalent symbols of the differential quadratic quantic

$$
d s^{2}=\sum_{i, k=1}^{n} a_{i k} d x_{i} d x_{k}
$$

These differential parameters and in particular the equations obtained by putting them equal to zero play an important rôle in all questions connected with the study of the orthogonality of directions in higher spaces.

In § 1 I set up four fundamental theorems concerning determinants, using ordinary (not symbolic) notation. In $\S 2$ the symbolic method is applied to the construction of four important formulas which are used in the sequel and furnish at the same time numerous relations between differential parameters. In $\S 3$ the directions orthogonal to all directions in a space of $\lambda$ dimensions given by the equations $U^{\prime}, \ldots, U^{n-\lambda}=$ const. are determined and $\S 4$ contains a general investigation of the conditions under which one space $V^{\prime}, \ldots, V^{n-\mu}=$ const. contains directions which are orthogonal to all directions of another space $U^{\prime}, \ldots, U^{n-\lambda}=$ const., and the determination of these directions.

\section{§1. Theorems on determinants.}

Designate the determinant $\left|x_{i}^{k}\right|$ of any $\mu^{2}$ quantities $x$, where $x_{i}^{k}$ is the element occupying the place in the $i$ th row and $k$ th column, by

$$
\left(x^{\prime} x^{2} \cdots x^{\mu}\right) \text {; }
$$

* Presented to the Society (Chicago) April 22, 1905. Received for publication September 30, 1905.

† The symbolio method has been explained by me in the paper $A$ symbolic treatment of the theory of invariants of quadratic differential quantics of $n$ variables, Transactions of the American Mathematical Society, vol. 4 (1903), pp. 441-469. I shall quote this paper by I. 
let us further use the abbreviation

$$
\left(x^{\prime} x^{2} \cdots x^{\lambda} u^{\prime} u^{2} \cdots\right)=\left(x^{\prime} x^{2} \cdots x^{\lambda} u\right),
$$

where the number of elements $u^{\prime}, u^{2}, \ldots$ is any.

1) If we form the determinant of degree $n+1$ the first $n$ rows of which are

$$
\begin{gathered}
a_{1}^{\prime} a_{1}^{2} \cdots a_{1}^{\lambda} u_{1}^{\prime} u_{1}^{2} \cdots, \\
a_{n}^{\prime} a_{n}^{2} \cdots \cdot a_{n}^{\lambda} u_{n}^{\prime} u_{n}^{2} \cdots,
\end{gathered}
$$

where the elements $a_{k}^{i}$ and $u_{k}^{i}$ are any arbitrary quantities, then the determinant will vanish if we take for the $n+1$ th row the elements

$$
\begin{array}{r}
\left(a^{\prime} b^{2} \cdots b^{\lambda-1} u\right),\left(a^{2} b^{2} \cdots b^{\lambda-1} u\right), \cdots,\left(a^{\lambda} b^{2} \cdots b^{\lambda-1} u\right), \\
\left(u^{\prime} b^{2} \cdots b^{\lambda-1} u\right),\left(u^{2} b^{2} \cdots b^{\lambda-1} u\right), \cdots,
\end{array}
$$

where again the quantities $b_{k}^{2}, \ldots, b_{k}^{\lambda-1}$ are any. But all the terms $\left(u^{k} b^{2} \cdots b^{\lambda-1} u\right)$ vanish. Thus, expanding the determinant according to its elements of the $n+1$ th row, we have

$$
\sum_{k=1}^{\lambda}(-1)^{k+1}\left(a^{k} b^{2} \cdots b^{\lambda-1} u\right)\left(a^{\prime} a^{2} \cdots a^{k-1} a^{k+1} \cdots a^{\lambda} u\right)=0,
$$

or, by putting

$$
\begin{aligned}
& a^{\lambda}=b^{\prime}, \\
& \sum_{k=1}^{\lambda-1}(-1)^{k+1}\left(a^{k} b^{2} \cdots b^{\lambda-1} u\right)\left(a^{\prime} a^{2} \cdots a^{k-1} a^{k+1} \cdots a^{\lambda-1} b^{\prime} u\right) \\
& =(-1)^{\lambda}\left(a^{\prime} a^{2} \cdots a^{\lambda-1} u\right)\left(b^{\prime} b^{2} \ldots b^{\lambda-1} u\right) \text {. }
\end{aligned}
$$

If finally we write $\lambda$ instead of $\lambda-1$ and place $b^{\prime}$ between $a^{k-1}$ and $a^{k+1}$, we obtain the formula

(1) $\sum_{k=1}^{\lambda}\left(a^{k} b^{2} \cdots b^{\lambda} u\right)\left(a^{\prime} a^{2} \cdots a^{k-1} b^{\prime} a^{k+1} \cdots a^{\lambda} u\right)=\left(a^{\prime} a^{2} \cdots a^{\lambda} u\right)\left(b^{\prime} b^{2} \cdots b^{\lambda} u\right)$.

This formula is identically true for all the involved quantities $a_{k}^{i}, b_{k}^{i}$ and $u_{k}^{i}$.

2) We extend formula (1) by putting $(\mu<\lambda)$

$$
a^{\mu+k}=c^{k} \quad(k=1, \cdots, \lambda-\mu),
$$

and performing the summation first from 1 to $\mu$, then from $\mu+1$ to $\lambda$. Thus we have

$$
\begin{aligned}
& \sum_{k=1}^{\mu}\left(a^{k} b^{2} \cdots b^{\lambda} u\right)\left(a^{\prime} \cdots a^{k-1} b^{\prime} a^{k+1} \cdots a^{\mu} c^{\prime} \cdots c^{\lambda-\mu} u\right) \\
& +\sum_{k=1}^{\lambda-\mu}\left(c^{k} b^{2} \cdots b^{\lambda} u\right)\left(a^{\prime} \cdots a^{\mu} c^{\prime} \cdots c^{k-1} b^{\prime} c^{k+1} \cdots c^{\lambda-\mu} u\right) \\
& =\left(a^{\prime} \cdots a^{\mu} c^{\prime} \cdots c^{\lambda-\mu} u\right)\left(b^{\prime} \cdots b^{\lambda} u\right)
\end{aligned}
$$


3) I wish to prove the following theorem

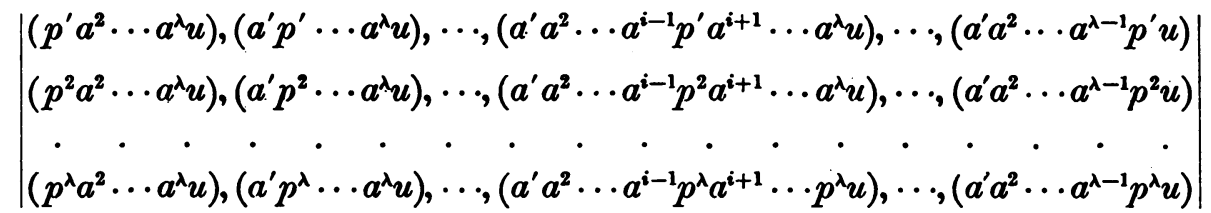

$$
\begin{aligned}
& =\left(p^{\prime} p^{2} \cdots p^{\lambda} u\right)\left(a^{\prime} a^{2} \cdots a^{\lambda} u\right)^{\lambda-1},
\end{aligned}
$$

where all the quantities $a_{k}^{i}, p_{k}^{i}, u_{k}^{i}$ are arbitrary.

We prove by induction from $\lambda-1$ to $\lambda$.

Every term of the minor $P^{k}$ of the term $\left(p^{k} a^{2} \ldots a^{\lambda} u\right)$ in the original determinant $\boldsymbol{P}$ contains the element $a^{\prime}$ which can be placed just in front of the elements $u$. The determinant $P^{k}$ is then of the same type as $P$, with $\lambda-1$ instead of $\lambda$, and $a^{\prime}, u$ instead of $u$, and since the theorem is supposed to be true for determinants of order $\lambda-1$, we have

or

$$
P^{k}=(-1)^{k+1+(\lambda-1)^{2}}\left(p^{\prime} \cdots p^{k-1} p^{k+1} \cdots p^{\lambda} a^{\prime} u\right)\left(a^{2} \cdots a^{\lambda} a^{\prime} u\right)^{\lambda-2},
$$

and

$$
P^{k}=\left(p^{\prime} \cdots p^{k-1} a^{\prime} p^{k+1} \cdots a^{\lambda} u\right)\left(a^{\prime} a^{2} \cdots a^{\lambda} u\right)^{\lambda-2},
$$

$$
P=\left(a^{\prime} a^{2} \cdots a^{\lambda} u\right)^{\lambda-2} \sum_{k=1}^{\lambda}\left(p^{k} a^{2} \cdots a^{\lambda} u\right)\left(p^{\prime} \cdots p^{k-1} a^{\prime} p^{k+1} \cdots p^{\lambda} u\right) .
$$

To this sum we can directly apply formula (1) and obtain

$$
P=\left(p^{\prime} \cdots p^{\lambda} u\right)\left(a^{\prime} \cdots a^{\lambda} u\right)^{\lambda-1} .
$$

Hence the theorem is generally true, since it is true for $\lambda=2$, the equation

$$
\left(p^{\prime} a^{2} u\right)\left(a^{\prime} p^{2} u\right)+\left(p^{2} a^{2} u\right)\left(p^{\prime} a^{\prime} u\right)=\left(p^{\prime} p^{2} u\right)\left(a^{\prime} a^{2} u\right)
$$

being a special case of $(1)$.

4) Let $\rho, v^{\prime}, v^{2}, \ldots, v^{\lambda}, u^{\prime}, u^{2}, \ldots, u^{n-\lambda}$ be any functions of the $n$ independent variables $x_{1}, x_{2}, \cdots, x_{n}$, and define further

$$
\rho_{k}=\frac{\partial \rho}{\partial x_{k}}, \quad v_{k}^{i}=\frac{\partial v^{i}}{\partial x_{k}}, \quad u_{k}^{i}=\frac{\partial u^{i}}{\partial x_{k}},
$$

then I wish to prove the following theorem on Jacobians

(4) $\left(\rho v^{\prime}, \rho v^{2}, \cdots, \rho v^{\lambda}, u\right)=\rho^{\lambda}\left(v^{\prime} v^{2} \cdots v^{\lambda} u\right)+\rho^{\lambda-1} \sum_{k=1}^{\lambda} v^{k}\left(v^{\prime} \cdots v^{k-1}, \rho, v^{k+1} \cdots v^{\lambda} u\right)$,

where on the left side the general element of any one of the first $\lambda$ columns is $\partial \rho v^{i} / \partial x_{k}$, the notation otherwise being the same as in the preceding work. We prove again by induction from $\lambda$ to $\lambda+1$. 
We have directly

$$
\left(\rho v^{\prime}, \cdots, \rho v^{\lambda}, \rho v^{\lambda+1}, u\right)=\rho\left(\rho v^{\prime}, \cdots, \rho v^{\lambda}, v^{\lambda+1}, u\right)+v^{\lambda+1}\left(\rho v^{\prime}, \cdots, \rho v^{\lambda}, \rho, u\right),
$$

but from (4), by putting the first one of the quantities $u$ equal to $v^{\lambda+1}$,

$$
\left(\rho v^{\prime}, \cdots, \rho v^{\lambda}, v^{\lambda+1}, u\right)=\rho^{\lambda}\left(v^{\prime} \cdots v^{\lambda+1} u\right)+\rho^{\lambda-1} \sum_{k=1}^{\lambda} v^{k}\left(v^{\prime} \cdots v^{k-1} \rho v^{k+1} \cdots v^{\lambda+1} u\right)
$$

and similarly

$$
\left(\rho v^{\prime}, \cdots, \rho v^{\lambda}, \rho, u\right)=\rho^{\lambda}\left(v^{\prime} \cdots v^{\lambda} \rho u\right)+\rho^{\lambda-1} \sum_{k=1}^{\lambda} v^{k}\left(v^{\prime} \cdots v^{k-1} \rho v^{k+1} \cdots v^{\lambda} \rho u\right) .
$$

Each one of the terms of the last sum vanishes, hence

$$
\begin{aligned}
\left(\rho v^{\prime}, \cdots \rho v^{\lambda+1}, u\right)=\rho^{\lambda+1}\left(v^{\prime} \cdots v^{\lambda+1} u\right)+\rho^{\lambda} \sum_{k=1}^{\lambda} v^{k}\left(v^{\prime} \cdots v^{k-1} \rho v^{k+1} \cdots v^{\lambda+1} u\right) & +\rho^{\lambda}\left(v^{\prime} \cdots v^{\lambda} \rho u\right),
\end{aligned}
$$

$$
\left(\rho v^{\prime}, \cdots, \rho v^{\lambda+1}, u\right)=\rho^{\lambda+1}\left(v^{\prime} \cdots v^{\lambda+1} u\right)+\rho^{\lambda} \sum_{k=1}^{\lambda+1} v^{k}\left(v^{\prime} \cdots v^{k-1} \rho v^{k+1} \cdots v^{\lambda+1} u\right) .
$$

The theorem is true for $\lambda=1$, namely

$$
(\rho v, u)=\rho^{\prime}(v, u)+\rho^{0} v(\rho, u),
$$

hence it is generally true.

\section{§2. Relations between differential parameters.}

5) In formula (2) we omit the quantities $u$, put $\lambda=n$ and write $\lambda$ instead of $\mu$. We let further $c^{\prime}, \ldots, c^{n-\lambda}$ be symbols of a quadratic differential quantic of $n$ variables and designate them by $f^{\prime}, \ldots, f^{n-\lambda}$. We finally multiply every term of $(2)$ by some alternating function $\left[f^{\prime}, \ldots, f^{n-\lambda}\right]=[f]$ of $f^{\prime}, \cdots, f^{n-\lambda}$. Then the general term of the second sum

$$
\begin{aligned}
& \left(f^{k} b^{2} \cdots b^{n}\right)\left(a^{\prime} \cdots a^{\lambda} f^{\prime} \cdots f^{k-} b^{\prime}, f^{+1} \cdots f^{n-\lambda}\right)[f] \\
& \quad=\left(f^{\prime} b^{2} \cdots b^{n}\right)\left(a^{\prime} \cdots a^{\lambda} b^{\prime} f^{2} \cdots f^{n-\lambda}\right)[f] .
\end{aligned}
$$

Hence all the terms of this sum are equal and we have

$$
\begin{aligned}
& \sum_{k=1}^{\lambda}\left(a^{k} b^{2} \cdots b^{n}\right)\left(a^{\prime} \cdots a^{k-1} b^{\prime} a^{k+1} \cdots a^{\lambda} f\right)[f]=\left(a^{\prime} \cdots a^{\lambda} f\right)\left(b^{\prime} \cdots b^{n}\right)[f] \\
& -(n-\lambda)\left(f^{\prime} b^{2} \cdots b^{n}\right)\left(a^{\prime} \cdots a^{\lambda} b^{\prime} f^{2} \cdots f^{n-\lambda}\right)[f] \text {. }
\end{aligned}
$$

take

$$
\begin{aligned}
& b^{\prime}=Q ; b^{2}, \ldots, b^{n}=\phi^{2}, \ldots, \phi^{n}(\text { symbols }), \\
& a^{\prime}, \ldots, a^{\lambda}=V^{\prime}, \ldots, V^{\lambda},
\end{aligned}
$$

$$
\left[f^{\prime}\right]=\left(U^{\prime} \ldots U^{\lambda} f^{\prime}\right)
$$


and multiply by $(P \phi)$, then the second term on the right side of (5) can be transformed by means of $I(34)$, namely

$$
\left(f^{\prime} \phi\right)(P \phi)\left(U^{\prime} \cdots U^{\lambda} f^{\prime} f^{2} \cdots f^{n-\lambda}\right)=(n-1) !\left(U^{\prime} \ldots U^{\lambda} P f\right),
$$

so that we obtain the following formula

$$
(P \phi)\left(U^{\prime} \ldots U^{\lambda} f\right) \sum_{k=1}^{\lambda}\left(V_{k} \phi\right)\left(V^{\prime} \ldots V^{k-1} Q V^{k+1} \ldots V^{\lambda} f\right)
$$

$=(P \phi)(Q \phi)\left(U^{\prime} \ldots U^{\lambda} f\right)\left(V^{\prime} \ldots V^{\lambda} f\right)$

7) If in (6) we put

$$
\begin{aligned}
& -(n-\lambda)(n-1) !\left(U^{\prime} \ldots U^{\lambda} P f\right)\left(V^{\prime} \ldots V^{\lambda} Q f\right) . \\
& Q=U^{\prime}=\psi \text { (symbol), }
\end{aligned}
$$

then we can apply to the first term on the right side the transformation I (34) so that

$$
(P \phi)(\psi \phi)\left(\psi U^{2} \ldots U^{\lambda} f\right)=(n-1) !\left(P U^{2} \ldots U^{\lambda} f^{\prime}\right) .
$$

The two terms on the right side of $(6)$ combine then to

$$
(n-\lambda+1)(n-1) !\left(P U^{2} \ldots U^{\lambda} f\right)\left(V^{\prime} \ldots V^{\lambda} f\right),
$$

and by writing $U^{\prime}$ instead of $P$ we obtain with a slight change the formula

$$
\begin{aligned}
(U \phi)\left(U^{2} \ldots U^{\lambda} f\right) & \sum_{k=1}^{\lambda}(-1)^{k+1}\left(V_{k} \phi\right)\left(V^{\prime} \ldots V^{k-1} V^{k+1} \ldots V^{\lambda} f\right) \\
& =(n-\lambda+1)(n-1) !\left(U^{\prime} \ldots U^{\lambda} f\right)\left(V^{\prime} \ldots V^{\prime} f\right) .
\end{aligned}
$$

8) I wish to prove that

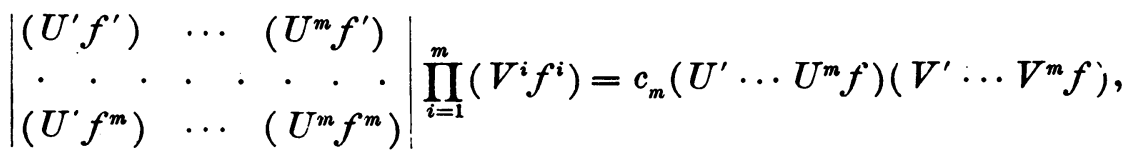

where every $f^{k}$ on the left side stands for a set of $n-1$ equivalent symbols and where $c_{m}$ is a numerical constant depending on $m$. Denote the left side of the above equation by $R$.

I prove by induction from $m-1$ to $m$. Then we have

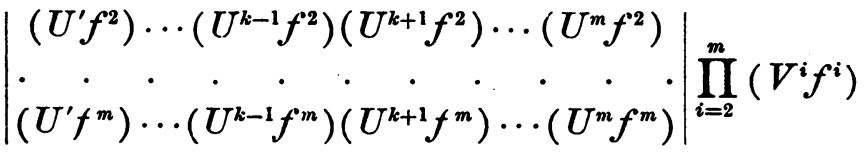

$$
\begin{aligned}
& =c_{m-1}\left(U^{\prime} \ldots U^{k-1}, U^{k+1} \ldots U^{m} f\right)\left(V^{2} \ldots V^{m} f\right),
\end{aligned}
$$


and if we develop the determinant-factor of $R$ according to its elements of the first row

$R=c_{m-1}\left(V^{\prime} f^{\prime}\right)\left(V^{2} \ldots V^{m} f\right) \sum_{k=1}^{m}(-1)^{k+1}\left(U^{k} f^{\prime}\right)\left(U^{\prime} \ldots U^{k-1} U^{k+1} \ldots U^{m} f\right)$,

which reduces by means of (7) to

$$
R=(n-m+1)(n-1) ! c_{m-1}\left(U^{\prime} \ldots U^{m} f\right)\left(V^{\prime} \ldots V^{m} f\right) .
$$

For $m=1$ the theorem is true, and $c_{1}=1$. Hence the theorem is generally true. The constant $c_{m}$ is easily determined, namely

so that

$$
c_{m}=(n-1)(n-2) \cdots(n-m+1)[(n-1) !]^{m-1},
$$

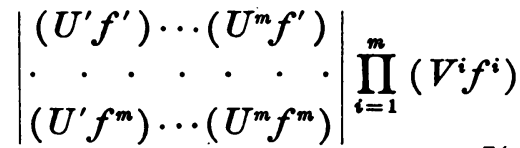

$$
\begin{aligned}
& =\frac{[(n-1) !]^{m}}{(n-m) !}\left(U^{\prime} \ldots U^{m} f\right)\left(V^{\prime} \ldots V^{m} f\right) .
\end{aligned}
$$

\section{§3. Determination of directions orthogonal to all directions in a given space $U$.}

We define in a general space $R_{n}$ of $n$ dimensions whose arc-element is determined by

$$
d s^{2}=\sum_{r, s=1}^{n} a_{r s} d x_{r} d x
$$

a surface (space) of $\lambda$ dimensions, $R_{\lambda}$, by the $n-\lambda$ equations

$$
U^{\prime}=\text { const., } \cdots, U^{n-\lambda}=\text { const., }
$$

where the $n-\lambda$ quantities $U$ are functions of $x_{1}, \ldots, x_{n}$.

The $n$ differentials $d x_{1}, \cdots, d x_{n}$ satisfying the $n-\lambda$ equations

$$
\sum_{r=1}^{n} U_{r}^{\prime} d x_{r}=0, \cdots, \sum_{r=1}^{n} U_{r}^{n-\lambda} d x_{r}=0,
$$

define a certain direction in $R_{\lambda}$. We agree, as always in the following, to denote differentiation with respect to $x_{r}$ by the lower index $r$.

In order to solve the equations. (11) symmetrically we introduce $\lambda$ arbitrary functions $V^{\prime}, \ldots, V^{\lambda}$ with the restriction, however, that the Jacobian

$$
D=\left(V^{\prime} \ldots V^{\lambda} U^{\prime} \ldots U^{n-\lambda}\right) \neq 0 .
$$


If the minors of the element $V_{r}^{k}$ in $D$ are denoted by $A^{k r}$, it is clear that the $\lambda$ systems of differentials

$$
d x_{1}^{k}=\rho A^{k 1}, \cdots, d x_{n}^{k}=\rho A^{k n}
$$$$
(k=1, \cdots, \lambda)
$$

satisfy equations (11) and represent $\lambda$ independent (i. e., not contained in a space of less than $\lambda$ dimensions) directions in $R_{\lambda}$. The general direction in $R_{\lambda}$ - general solution of $(11)$ - is then given by

$$
d x_{1}=\sum_{k=1}^{\lambda} \rho_{k} A^{k 1}, \ldots, d x_{n}=\sum_{k=1}^{n} \rho_{k} A^{k n},
$$

where $\rho_{1}, \ldots, \rho_{\lambda}$ are arbitrary parameters.

If $p$ is any arbitrary quantity we have

$$
\sum_{r=1}^{n} p_{r} d x_{r}=\sum_{k=1}^{\lambda} \rho_{k}\left(V^{\prime} \ldots V^{k-1} p V^{k+1} \ldots V^{\lambda} U\right),
$$

and this expression serves conveniently to define the direction $d x_{1}, \ldots, d x_{n}$ as the ratios of the coefficients of $p_{1}, \cdots, p_{r}$. We shall simply call it the direction $p$.

The condition that two directions defined by two systems $d x$ and $\delta x$ are to be perpendicular to each other in $R_{n}$ is

or, in symbolic notation,

$$
\sum_{r, s=1}^{n} a_{r s} d x_{r} \delta x_{s}=0,
$$

$$
\sum_{r=1}^{n} f_{r} d x_{r} \cdot \sum_{s=1}^{n} f_{c} d x_{\odot}=0 \text {. }
$$

If now the direction $\delta x$ in $R_{n}$ is to be perpendicular to all the directions $d x$ in $R_{n}$ it will be necessary and sufficient that $\delta x$ is perpendicular to the $\lambda$ independent directions defined by (13).

Hence

$$
\left(V^{\prime} \ldots V^{k-1} f V^{k+1} \ldots V^{\lambda} U\right) \sum_{s=1}^{n} f_{s} \delta x^{s}=0
$$

for every $k=1, \ldots, \lambda$.

To solve these equations we adjoin again $n-\lambda$ arbitrary functions $W^{\prime}, \ldots, W^{n-\lambda}$ and form the determinant $\Delta$ of the derivatives of the quantities $W$ with respect to $x_{1}, \cdots, x_{n}$ and the coefficients of the differentials $\delta x_{e}$ in (17). We obtain

$$
\Delta=\left(W^{\prime} \ldots W^{n-\lambda} f^{\prime} \cdots f^{\lambda}\right) \prod_{k=1}^{n}\left(V^{\prime} \ldots V^{k-1} f V^{k+1} \ldots V^{\lambda} U\right)
$$

and if we permute the equivalent symbols $f^{\prime}, \cdots, f^{\lambda}$ in all possible ways, add together and divide by $\lambda !$, 
$\Delta=\frac{1}{\lambda !}\left(f^{\prime} \cdots f^{\lambda} W\right)$

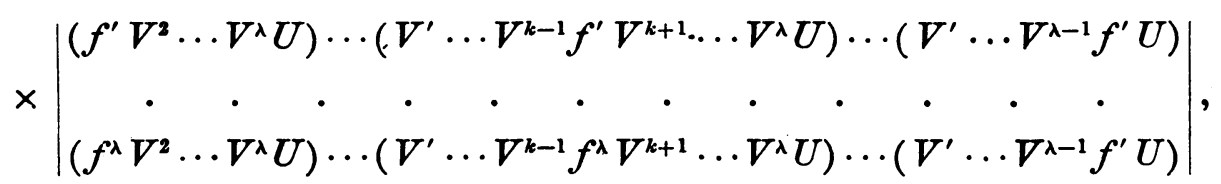

which reduces according to $(3)$ to

$$
\Delta=\frac{1}{\lambda !}\left(f^{\prime} \ldots f^{\lambda} W\right)\left(f^{\prime} \ldots f^{\lambda} U\right)\left(V^{\lambda} \ldots V^{\lambda} U\right)^{\lambda-1} .
$$

Denoting now the minor of the element $W_{r}^{a}$ in $\Delta$ by $B^{a r}$ we find the following $n-\lambda$ systems of solutions of (17) by taking the factor

$$
\frac{1}{\lambda !}\left(V^{\prime} \ldots V^{\lambda} U\right)^{\lambda-1}
$$

into the proportionality-factor $\sigma$,

$$
\delta x_{1}^{a}=\sigma B^{a 1}\left(f^{\prime} \cdots f^{\lambda} U\right), \cdots, \delta x_{n}^{a}=\sigma B^{a n}\left(f^{\prime} \cdots f^{\lambda} U\right) \quad(a=1, \cdots, n-\lambda) .
$$

The general expression of any direction $\delta x$ in $R_{n}$ perpendicular to every direction in $R_{\lambda}$ is therefore given by

or, using the notation explained in (15),

$$
\delta x_{r}=\left(f^{\prime} \cdots f^{\lambda} U\right) \sum_{a=1}^{n-\lambda} \sigma_{a} B^{a r} \quad(r=1, \cdots, n),
$$

$$
\sum_{r=1}^{n} q_{r} \delta x_{r}=\left(U^{\prime} \ldots U^{n-\lambda} f\right) \sum_{a=1}^{n-\lambda} \sigma_{a}\left(W^{\prime} \ldots W^{a-1} q W^{a+1} \ldots W^{n-\lambda} f\right),
$$

where $\sigma_{1} \cdots \sigma_{n-\lambda}$ are $n-\lambda$ arbitrary parameters.

By a proper selection of these parameters this expression can be considerably simplified. I put

$$
\sigma_{a}=\left(W^{a} \phi\right)\left(U^{k} \phi\right),
$$

where the $n-1$ quantities $\phi$ are again symbols of (9).

To (19) we can directly apply formula (6) where now the term

$$
\left(U^{\prime} \ldots U^{n-\lambda} P f\right)
$$

vanishes for $P=U^{k}$ so that we have

$$
\sum_{r=1}^{n} q_{r} d x_{r}=(q \phi)\left(U^{k} \phi\right)\left(U^{\prime} \ldots U^{n-\lambda} f\right)\left(W^{\prime} \ldots W^{n-\lambda} f\right),
$$

and since we are only concerned with the ratios of the differentials $d x$ we can omit the factor

$$
\left(U^{\prime} \ldots U^{n-\lambda} f\right)\left(W^{\prime} \ldots W^{n-\lambda} f\right)
$$


which admits of actual (not only symbolical) interpretation and have now the result

$$
\sum_{r=1}^{n} q_{r} \delta x_{r}^{k}=\rho\left(U^{k} f\right)(q f) \quad(k=1, \cdots, n-\lambda),
$$

which defines $n-\lambda$ independent directions in $R_{n}$, all of which are perpendicular to the general direction $p(15)$ in $R_{\lambda}$. From (20) we form the general direction in $R_{n}$ perpendicular to $R_{\lambda}$, namely,

$$
\sum_{r=1}^{n} q_{r} \delta x_{r}=\sum_{k=1}^{n-\lambda} \rho_{k}\left(U^{k} f\right)(q f)
$$

where $\rho_{1}, \ldots, \rho_{n-\lambda}$ are arbitrary parameters. Thus we have the following

Theorem I. Given a space of $\lambda$ dimensions $R_{\lambda}$ by the $n-\lambda$ equations

$$
U^{\prime}=\text { const., } \cdots, U^{n-\lambda}=\text { const., }
$$

contained in the general space $R_{n}$ of $n$ dimensions defined by (9). Then every direction $\delta x_{1}: \delta x_{2}: \cdots: \delta x_{n}$ in $R_{n}$ which is normal at a point $P$ of $R_{\lambda}$ to every possible direction in $R_{\lambda}$ at $P$ is given by the ratios of the coefficients $q_{1}: q_{2}: \cdots: q_{n}$ in the expression (21).

A posteriori it is easily verified that the values of $\delta x$ taken from (21) or (20) satisfy equation (17), for

$$
\sum_{r=1}^{n} f_{r} \delta x_{r}^{i}=\rho\left(U^{i} \phi\right)(f \phi),
$$

and $\left(U^{i} \phi\right)(f \phi)\left(V^{\prime} \ldots V^{k-1} f V^{k+1} \ldots V^{\lambda} U\right)=0$ from $I(34)$ for every value of $i$ and $k$.

§4. General study of the case where one space $V$ contains directions orthogonal to all directions of another space $U$.

If a space $V$ of $\mu$ dimensions

$$
V^{\prime}, V^{2}, \cdots, V^{n-\mu}=\text { const. }
$$

contains a direction which is normal to all the directions of a space $U$ of $\lambda$ dimensions

$$
U^{\prime}, U^{2}, \cdots, U^{n-\lambda}=\text { const., }
$$

then the $n-\mu$ equations

$$
\sum_{r=1}^{n} V_{r}^{i} d x_{r}=0 \quad(i=1, \cdots, n-\mu)
$$

must be satisfied by substituting for $d x_{1}, \cdots d x_{n}$ the coefficients of $q$ of the expression

$$
(q \phi) \sum_{k=1}^{n-\lambda} \phi_{k}\left(U^{k} \phi\right),
$$


i. e., we must have

$$
\left(V^{i} \phi\right) \sum_{k=1}^{n-\lambda} \rho_{k}\left(L^{\top i} \phi\right)=0 \quad(i=1, \cdots, n-\mu) .
$$

Every system of values $\rho_{k}$ satisfying these $n-\mu$ homogeneous linear equations gives us one direction $q$ of the required property.

We treat first the case where the number of equations is less than the number of unknowns $\rho_{k}$, i. e.,

$$
n-\mu<n-\lambda \quad \text { or } \quad \mu>\lambda .
$$

Then the general solution of $(25)$ is

$$
\rho_{k}=(-1)^{k+1}\left(U^{\prime} \ldots U^{k-1} U^{k+1} \ldots U^{n-\lambda} f\right)\left(V^{\prime} \ldots V^{n-\mu} \omega^{\prime} \ldots \omega^{\mu-\lambda-1} f\right)
$$

where $\omega^{\prime}, \ldots, \omega^{\mu-\lambda-1}$ are arbitrary quantities. Indeed the left side of (25) reduces by means of $(7)$ to

$$
\left(U^{\prime} \ldots U^{n-\lambda} f\right)\left(V^{i} V^{\prime} \ldots V^{n-\mu} \omega^{\prime} \cdots \omega^{\mu-\lambda-1} f\right),
$$

which vanishes for $i=1, \cdots, n-\mu$.

We obtain the direction $q$ itself by substituting the value of $\rho_{k}(26)$ into (24) which gives, again according to (7),

$$
\left(q V^{\prime} \ldots V^{n-\mu} \omega \cdots \omega^{\mu-\lambda-1} f\right)\left(U^{\prime} \ldots U^{n-\lambda} f\right) \text {. }
$$

Hence we have

Theorem II. If a space $U$ defined by $U^{\prime}, \ldots, U^{n-\lambda}=$ const., and a space $V$ defined by $V^{\prime}, \cdots, V^{n-\mu}=$ const., have at least one point $P$ in common, then there exists, provided that $\mu>\lambda$, at every point $P$ always and in general (i. e., if no special relations between $U$ and $V$ hold) only $\infty^{\mu-\lambda-1}$ (i. e., $\mu-\lambda$ independent) directions in $V$ which are perpendicular to all directions in $U$ through $P$. These directions are given by the coefficients of $q_{1} \cdots q_{n}$ in the expression (27).

If there shall exist more than $\mu-\lambda$ independent directions in $V$ normal to $U$, then the rank* of the matrix of the coefficients of $\rho_{k}$ in (25) must be $<n-\mu$. If the rank is $n-\mu-s$, then there are $\mu-\lambda+s$ independent directions. Putting in this case for abbreviation

$$
n-\mu-s+1=\alpha,
$$

we see that every determinant

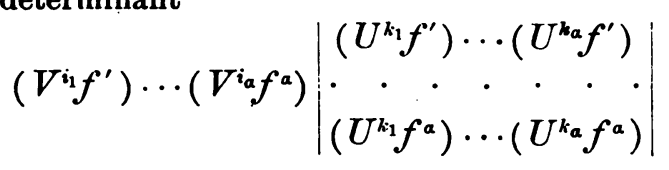

* A matrix is of rank $r$ if all its determinants of degree $r+1$ vanish, but not all its determinants of degree $r$. 
must vanish for all values $i_{1}, \cdots, i_{\alpha}=1, \cdots, n-\mu$, and $k_{1}, \cdots, k_{\alpha}=1, \ldots n-\lambda$. Applying now to this determinant formula (8) we have the conditions

$$
\left(U^{k_{1}} \ldots U^{k_{a}} f\right)\left(V^{k_{1}} \ldots V^{i_{a}} f\right)=0 .
$$

If these conditions are satisfied the quantities $\rho_{k}$ are determined by any (properly chosen) $n-\mu-s$ of the equations (25). We find

$$
\rho_{k}=(-1)^{k+1}\left(U^{\prime} \cdots U^{k-1} U^{k+1} \cdots U^{n-\lambda} f\right)\left(V^{i_{1}} \ldots V^{i_{a-1}} \omega^{\prime} \cdots \omega^{n-\lambda-a} f^{\prime}\right)
$$

and the required $\mu-\lambda+s$ independent directions as the coefficients of $q_{1}, \cdots, q_{n}$ in

$$
\left(q V^{i_{1}} \ldots V^{i_{\alpha-1}} \omega^{\prime} \ldots \omega^{\mu-\lambda+8-1} f\right)\left(U^{\prime} \ldots U^{n-\lambda} f\right) .
$$

We have, then, the following

TheоRem III. If a space $V(22)$ and $U(23)$, where $\mu>\lambda$, have at least one point $P$ in common, then the necessary and sufficient conditions that $V$ at every point $P$ contains $\mu-\lambda+s$ independent directions which are perpendicular to all directions $U$ through $P$ are

$$
\left(U^{k_{1}} \ldots U^{k_{a}} f\right)\left(V^{i_{1}} \ldots V^{i_{a}} f\right)=0,
$$

for every set of values

and

$$
k_{1}, \cdots, k_{a}=1, \cdots, n-\lambda \text {, }
$$

where

$$
i_{1}, \cdots, i_{a}=1, \ldots, n-\mu \text {, }
$$

$$
\alpha=n-\mu-s+1 \text {. }
$$

These directions are given by the coefficients of $q_{1}, \cdots, q_{n}$ in

$$
\left(q V^{i_{1}} \ldots V^{i_{n-\mu-\imath} \omega^{\prime}} \ldots \omega^{\mu-\lambda+8-1} f\right)\left(U^{\prime} \ldots U^{n-\lambda} f\right) .
$$

Applying now similar methods to the discussion of the remaining cases $\mu=\lambda$ and $\mu<\lambda$, always using equations (7) and (8) for reduction, we are led to the following theorems.

Theorem IV. If two spaces of equal dimensions $U^{\prime}, \cdots, U^{n-\lambda}=$ const., and $V^{\prime}, \ldots, \nabla^{n-\lambda}=$ const. have at least one point in common, then the necessary and sufficient condition that $V$ contains at every point $\boldsymbol{P}$ one direction normal to all directions in $U$ through $P$ is

$$
\left(U^{\prime} \cdots U^{n-\lambda} f\right)\left(V^{\prime} \ldots V^{n-\lambda} f\right)=0 .
$$

This direction is given by the coefficients of $q_{1} \cdots q_{n}$ in

$$
\left(q V^{\prime} \ldots V^{k-1} V^{k-1} \ldots V^{n-\lambda} f\right)\left(U^{\prime} \ldots U^{n-\lambda} f\right),
$$


where $k$ is arbitrary. In this case there is also one direction in $U$ which is normal to every direction in $V$; it is given by

$$
\left(q U^{\prime} \ldots U^{k-1} U^{k+1} \ldots U^{n-\lambda} f\right)\left(V^{\prime} \ldots V^{n-\lambda} f\right) .
$$

Theorem V. If a space $\nabla(22)$ and $U(23)$, where $\dot{\mu} \leqq \lambda$, have at least one point, $P$ in common, then the necessary and sufficient conditions that $V$ at every point $\boldsymbol{P}$ contains $s$ independent directions which are normal to all directions in $U$ through $P$ are

$$
\left(V^{i_{1}} \ldots V^{i_{\beta}} f\right)\left(U^{k_{1}} \ldots U^{k \beta} f\right)=0
$$

for every set of values

where

$$
\begin{aligned}
& i_{1}, \cdots, i_{\beta}=1, \cdots, n-\mu, \\
& k_{1}, \cdots, k_{\beta}=1, \cdots, n-\lambda,
\end{aligned}
$$

$$
\beta=n-\lambda-s+1 \text {. }
$$

These directions are given by the coefficients of $q_{1} \cdots q_{n}$ in the expression

$$
\left(q V^{i_{1}} \ldots V^{i_{n-\lambda-s} \omega^{\prime}} \ldots \omega^{s-1} f\right)\left(U^{\prime} \ldots U^{n-\lambda} f\right) .
$$

The University of Chicago,

September, 1905. 